\title{
Responses of blood vessels in the rabbit knee to acute joint inflammation
}

\author{
A Khoshbaten, W R Ferrell
}

\begin{abstract}
These experiments examined the responses of articular blood vessels in the rabbit knee to induction of acute joint inflammation by intraarticular injection of $4 \%$ kaolin suspension. This produced an inflammatory response, which became evident about four hours after injection. Electrical stimulation of the nerve supply to the knee joint before induction of inflammation produced a biphasic responsean initial vasoconstriction during the stimulation phase followed by dilatation after stimulation stopped. These responses were followed up for eight hours and it was noted that the constrictor response became progressively greater, producing a further $19 \%$ decrease in blood flow during nerve stimulation about three hours after the injection of kaolin. The sensitivity of postjunctional $\alpha$ adrenoceptors, however, showed still greater increase in the inflamed joint as close intraarterial injection of $10^{-6} \mathrm{M}$ adrenaline produced an additional $30 \%$ reduction in blood flow four hours after kaolin injection compared with the control response. Possibly, the smaller enhancement of the constrictor response to nerve stimulation in the inflamed joint may reflect sensitisation of prejunctional $\alpha$ adrenoceptors in addition to the effects exerted on postjunctional $\alpha$ adrenoceptors by the inflammatory process. The dilator response also increased over eight hours, though this rise was less marked. These findings indicate that even over the limited time span of the experiments, significant alterations occurred in factors which influence the calibre of articular blood vessels.
\end{abstract}

The factors regulating the calibre of articular blood vessels are at present poorly understood. It has been shown that the blood vessels supplying the knee joint of the dog are innervated by sympathetic efferent fibres, which when stimulated produce vasoconstriction. ${ }^{1}$ More recently it was shown that electrical stimulation of the posterior articular nerve supplying the dorsal aspect of the cat knee joint produced a biphasic response-an initial vasoconstriction during stimulation followed by a longlasting dilatation. ${ }^{2}$ The dilator response seemed to be mediated by substance $P$ release from unmyelinated joint afferent fibres as it was inhibited by intra-articular injection of the potent substance $\mathrm{P}$ antagonist d-Pro, ${ }^{4}$ $\mathrm{d}-\mathrm{Trp}^{7}{ }^{10}-\mathrm{SP}(4-11) .^{2}$ The constrictor response is likely to be mediated via $\alpha_{1}$ adrenoceptors as these are the principal type of postjunctional receptor found on rabbit articular blood vessels. ${ }^{3}$ Adrenaline and noradrenaline were equally effective in eliciting vasoconstriction in these vessels, which also appear to be devoid of $\beta$ adrenoceptors. $^{3}$

These experiments examined the possibility that the response to electrical stimulation of the nerve supply to the rabbit knee joint is altered by experimentally induced acute joint inflammation. This was carried out to determine whether the vasodilatation known to occur in joint inflammation might be attributed to alteration of neurotransmitter release at sympathetic nerve endings or change in adrenoceptor sensitivity induced by the inflammatory process.

\section{Materials and methods}

Adult New Zealand rabbits weighing about $3 \mathrm{~kg}$ were anaesthetised with a mixture of diazepam $(0.5 \mathrm{mg} / \mathrm{kg}$ intraperitoneally) and Hypnorm (0.1 $\mathrm{mg} / \mathrm{kg}$ intramuscularly). Thereafter, anaesthesia was maintained with a gaseous mixture of $\mathrm{O}_{2} / \mathrm{N}_{2} \mathrm{O} /$ halothane (2\%), which was delivered via a tracheal cannula. The common carotid artery was cannulated to permit monitoring of arterial blood pressure. The anteromedial aspect of the knee was exposed, the patellar ligament sectioned close to the patella, and the underlying articular tissue carefully exposed (fig 1). A 21G hypodermic thermocouple was inserted into the joint cavity and connected to an amplifier (Thermalert TH-6D) to provide a measure of intra-articular temperature. Relative changes in blood flow were measured with a near-infrared (780 $\mathrm{nm}$ ) laser Doppler flowmeter (Moor Instruments MBF2). The laser Doppler technique has been shown previously to provide a means of assessing synovial blood flow in the human knee. ${ }^{4}$ The laser probe was placed on the surface of the capsule and the surrounding tissue covered with cling film. The signal from the flowmeter was recorded on one channel of a pen recorder with blood pressure being recorded on the other channel.

The saphenous artery was cannulated in a retrograde direction with a 25G polythene cannula, which was advanced until the tip was just below the branches supplying the knee joint. Other branches were ligated. The saphenous nerve was dissected free close to the groin, the proximal end cut, and all branches except those to the knee were sectioned. Bipolar silver wire electrodes were then placed on the distal end of the nerve, and the stimulator (Harvard Advanced stimulator) was set to deliver $30 \mathrm{~s}$ trains of retangular pulses of $1 \mathrm{~ms}$ width, 10 $\mathrm{Hz}$ frequency, and $10 \mathrm{~V}$ amplitude. 


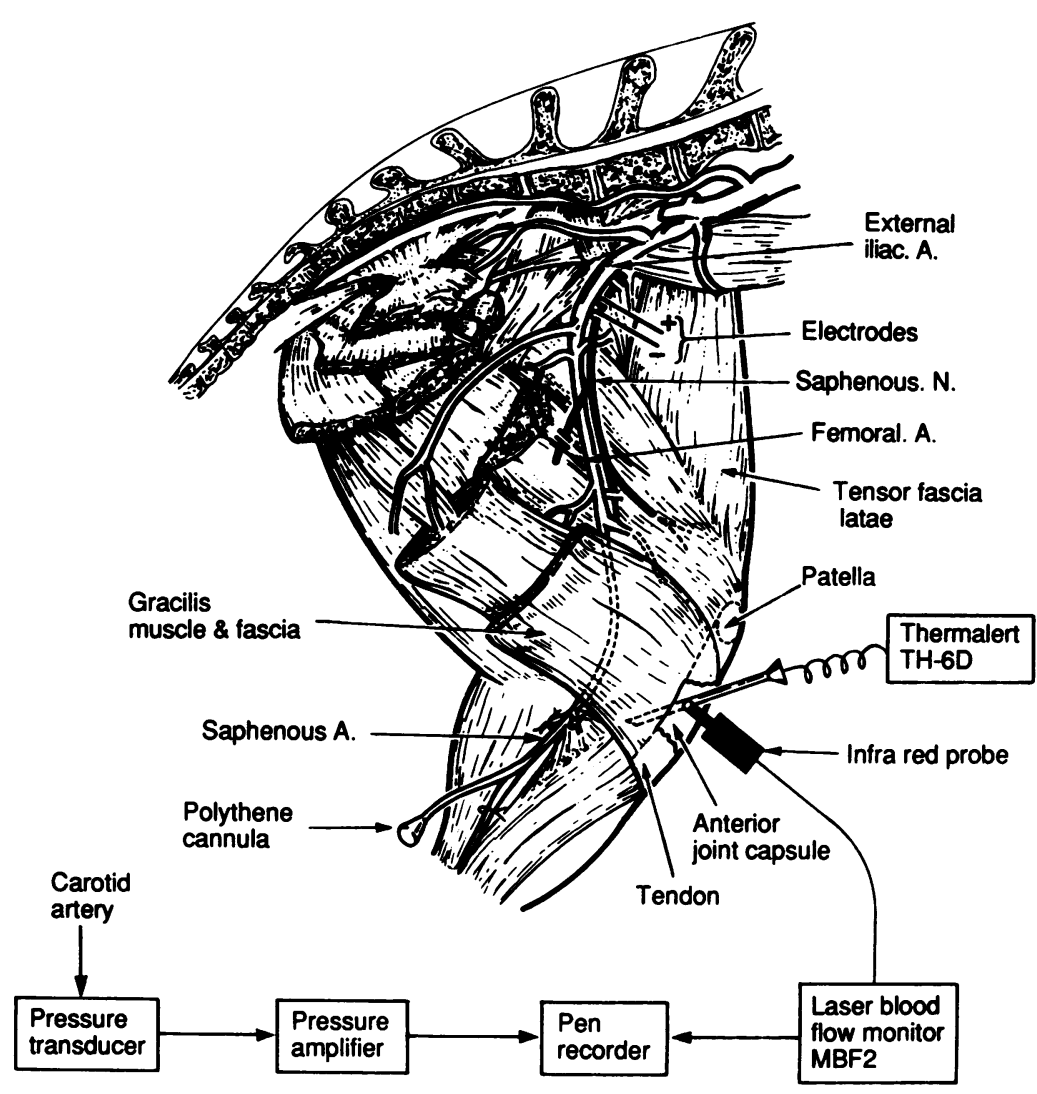

Figure 1: Diagram of the experimental arrangement. View of the medial aspect of the rabbit knee.
Simultaneous monitoring of the intra-articular temperatures of the two knees from the same animal showed characteristic responses. The knee that was injected with saline showed a progressive fall in intra-articular temperature with time (fig 3A), presumably owing to cooling of the immobile knee. The knee injected with kaolin also showed a fall in temperature initially, but this eventually levelled out (fig $3 \mathrm{~A}$ ). There was a $2^{\circ} \mathrm{C}$ difference in the temperature before injection because the kaolin injected knee was exposed to the atmosphere to obtain the blood flow measurements. It is noticeable that the initial temperature difference between the knees was almost completely reversed by the end of eight hours. This suggests that the kaolin injected knee was becoming inflamed by this time. Measurement of Evans blue extravasation in the two knees of five rabbits showed significant inflammation in the kaolin treated side compared with the saline injected side (fig 3B).

The responses to nerve stimulation were monitored in two groups of animals every half hour over a period of seven to eight hours. In one group of animals the knee was injected with saline $(n=3)$, while in the other group $(n=5)$ the knee was injected with the kaolin suspension. As shown in fig 4A, before kaolin injection, nerve stimulation resulted in a $30 \%$ reduction in blood flow. This constrictor response to nerve stimulation increased in amplitude after kaolin injection, producing a further $19 \%$ reduction of blood flow some three hours after injection, which fluctuated thereafter but always remained greater than the constrictor response in the saline injected knees. It was noticeable that the constrictor response in the saline injected knees articular injection of $4 \%$ kaolin suspension (Sigma) into one knee joint while the other was injected with sterile physiological saline to act as an internal control. The responses of the knees were examined by monitoring intra-articular temperature and by measurement of Evans blue extravasation into the joint tissue of the two knees. As Evans blue binds to plasma proteins normally restricted to the vascular compartment its presence in joint tissues provides an indirect means of assessing capillary permeability. The technique used to extract Evans blue and its subsequent spectrophotometric measurement has been described previously. ${ }^{5}$ Evans blue 75 $\mathrm{mg} / \mathrm{kg}$ was injected intravenously before intraarticular injection and the tissue samples were obtained at the end of the experimental period (about eight hours after intra-articular injection of saline or carrageenan).

Unless otherwise stated, data points in graphs represent means (SEM). Statistical analysis was by paired or unpaired $t$ test, as appropriate.

\section{Results}

Electrical stimulation of the saphenous nerve produced a repeatable response consisting of reduction in blood flow during the period of nerve stimulation, indicating vasoconstriction, followed by a longer lasting dilator response (fig 2). This response to nerve stimulation remained relatively stable and reproducible upon repeated stimulation, as long as the $30 \mathrm{~s}$ pulse trains were not reapplied until five minutes had elapsed from the previous period of stimulation.

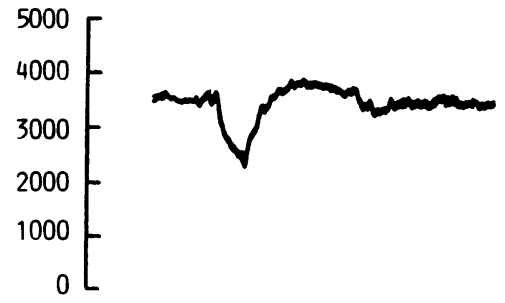

Figure 2: The top trace shows the signal obtained from the blood flow monitor in response to electrical stimulation of the saphenous nerve. A reduction in blood flow occurs during stimulation followed by dilatation. As the nerve was sectioned proximally heart rate and blood pressure remained constant. 

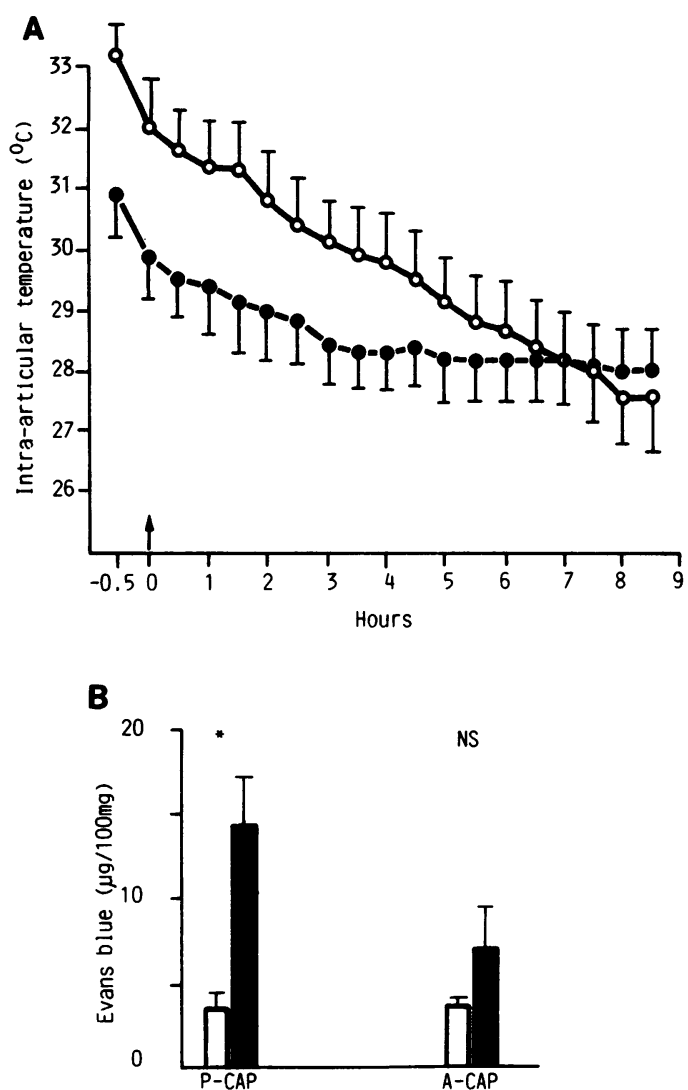

Figure 3: (A) Change in intra-articular temperature in knees injected with saline $(O)$ compared with those injected with $4 \%$ kaolin suspension (O). The arrow indicates the point at which injections were given. Results are means (SEM); $n=5$ in both cases. (B) Comparison of Evans blue content of the joint capsule in saline injected knees (open histogram) with knees injected with $4 \%$ kaolin (filled histogram). A significant difference in Evans blue content was found in the posterior capsule $(P-C A P)$. Although Evans blue content was also increased in the anterior capsule $(A-C A P)$ in the kaoline injected knee compared with control, this was not significant. Means $(S E M) ; n=5 ;{ }^{*} p<0 \cdot 05$.

was also enhanced, but to a lesser extent ( $9 \%$ maximum).

The dilator response to nerve stimulation showed less obvious change with time (fig 4B). Although the mean value of the kaolin injected knee peaked at four hours, it was not statistically significantly different from the value obtained from saline injected knees at this time. Comparison of the magnitude of the dilator response to nerve stimulation before injection of kaolin with the values obtained after injection, however, showed that at six hours the dilator response was significantly greater than control $(p<0.05)$ and by seven hours the difference was greater still $(p<0.001)$. In the saline injected knees, although some variation in the dilator response occurred over the seven hour period, none of the values differed significantly from the control (before saline injection) value. This difference may be partly attributable to the control value for the kaolin injected knees being less than that for the saline injected knees and partly due to the greater variability (as shown by the standard error bars) of the control values in the saline injected knees compared with the kaolin injected knees. Although the means of the control values differed between the two
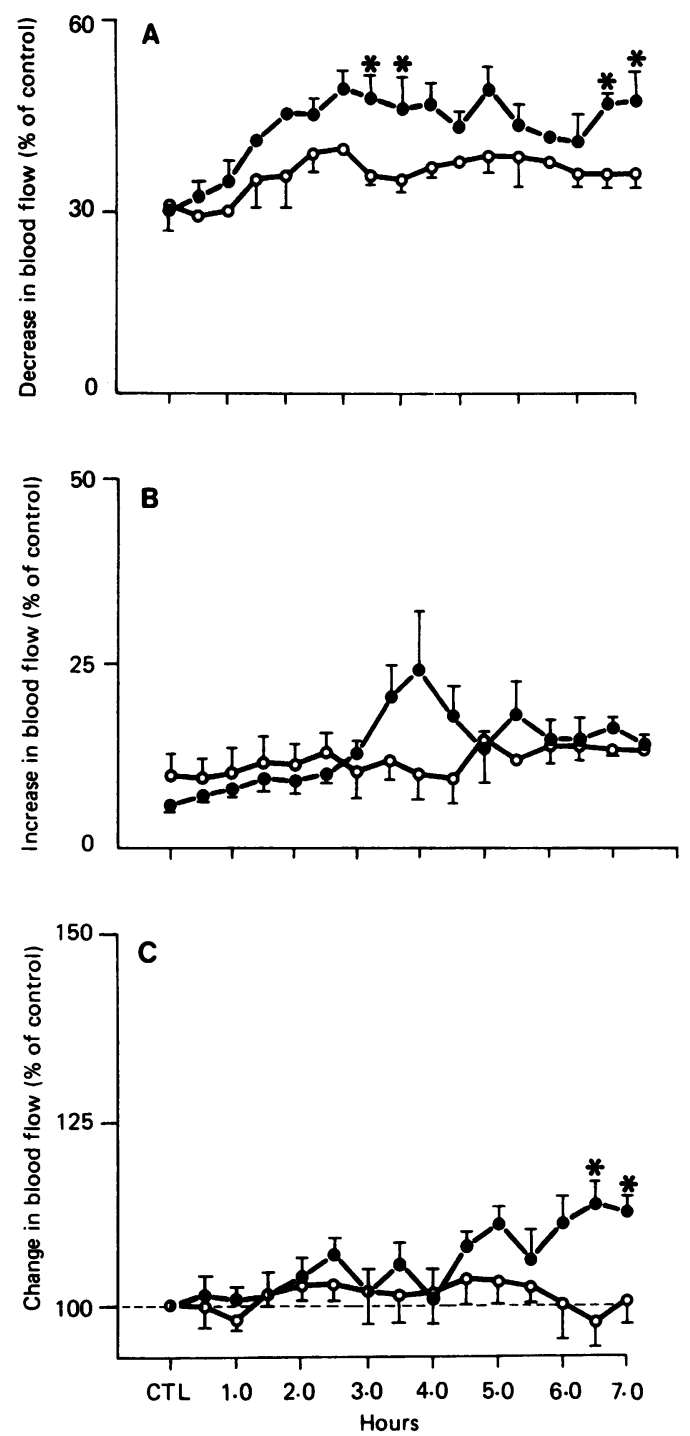

Figure 4: (A) Effect of intra-articular kaolin injection (O) and saline injection $(O)$ on the magnitude of the constrictor response to nerve stimulation over eight hours. Change in blood flow is expressed as percentage decrease in blood flow compared with baseline value immediately before

stimulation. $C T L$ represents the response to nerve stimulation immediately before injection of kaolin or saline. (B) Effect of intra-articular kaolin injection (O) and saline injection (O) on the magnitude of the dilator response after nerve stimulation. In this case the change in blood flow is expressed as percentage increase in flow relative to the value obtained immediately before nerve stimulation. CTL represents the dilator response obtained before injection of kaolin or saline. (C) Measurement of change in blood flow in kaolin injected knees (O) compared with saline injected knees (O) over seven hours. Change in blood flow is relative to the control value (CTL) obtained immediately after injection of these substances. Means (SEM); $n=5 ;{ }^{*} p<0.05$.

knees, this difference was not statistically significant.

Even though the joint capsule was exposed to the external environment for many hours, blood flow changed little over this time in the saline injected knee (fig 4C). In the kaolin injected knee little change in blood flow occurred until about $61 / 2$ hours after injection when statistically significant differences in blood flow occurred compared with the saline injected knee. When compared with the control value the kaolin injected knees showed consistently significant 


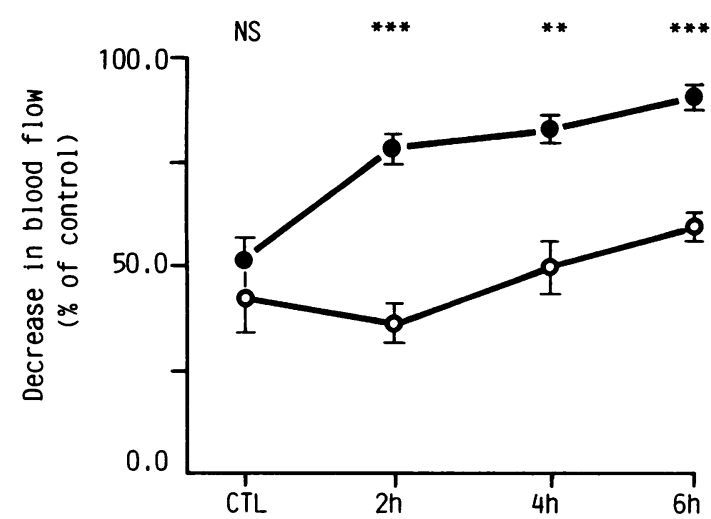

Figure 5: Response to close intra-arterial injection of $10^{-6} \mathrm{M}$ adrenaline in animals with kaolin injected knees. ( ; $n=6)$ compared with the response obtained in animals with saline injected knees $(\mathrm{O} ; n=3)$. Immediately after injection of these substances (CTL) the means do not differ significantly. After two hours the responses in the kaolin injected knees are greater and remain increased thereafter compared with saline injected knees. ${ }^{* * *} p<0.001 ;{ }^{* *} p<0.01$.

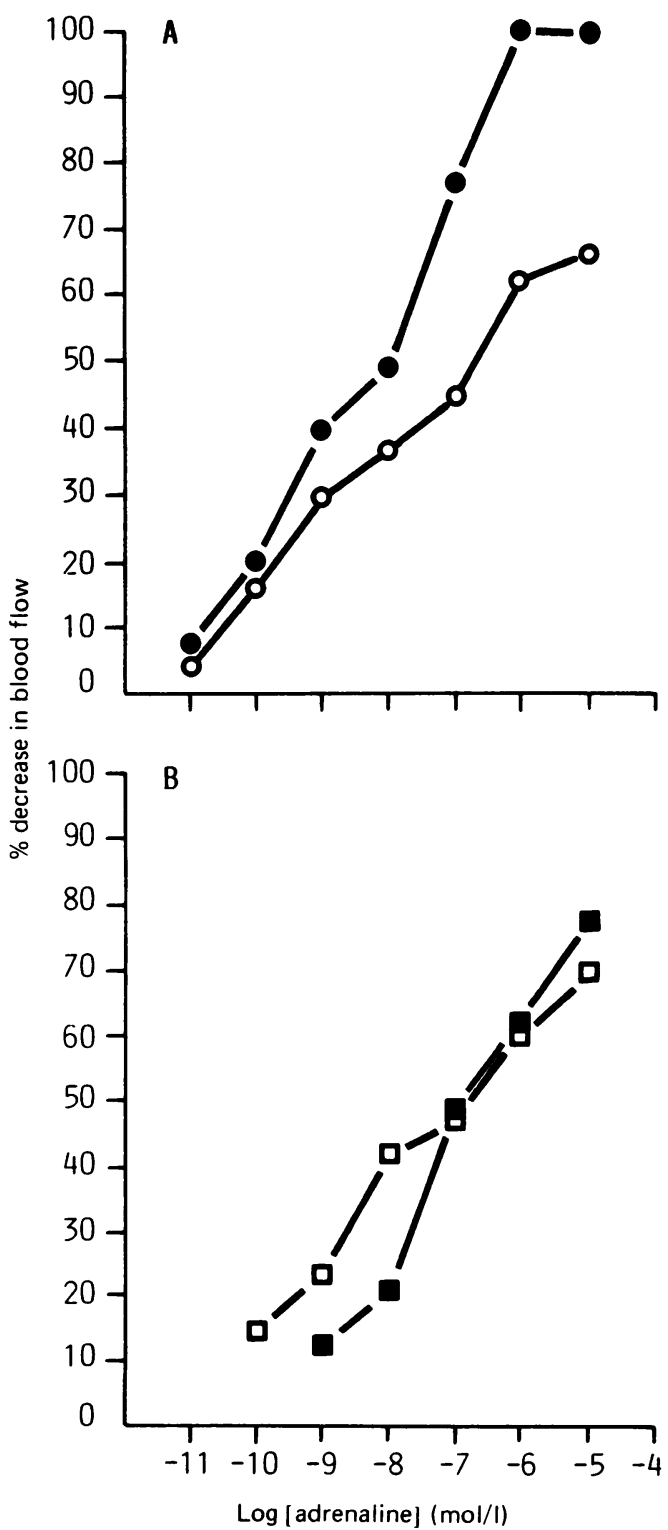

Figure 6: (A) Comparison of the dose-response relation to close intra-arterial injection of adrenaline in kaolin injected knees $(O)$ and saline injected knees $(O)$ eight hours after intra-articular injection of these substances.

(B) Dose-response relation to close intra-arterial injection of adrenaline in saline injected knee immediately after injection $(\square)$ and eight hours after $(\square)$ in the same animal. differences at $4 \frac{1}{2}$ hours and beyond, whereas none of the values differed significantly from control in the saline injected knees.

Although the responses to nerve stimulation were modestly enhanced by the inflammatory process, larger changes occurred in the sensitivity of the $\alpha$ adrenoceptors as the response to close intra-arterial injection of adrenaline $\left(10^{-6}\right.$ mol/l) was modified (fig 5). Immediately after intra-articular injection of saline or kaolin there was little difference in the constriction induced by adrenaline, which at this dose produced about a $50 \%$ reduction in flow. When the same dose of adrenaline was given two hours later, however, a highly significant rise in the response occurred in kaolin injected knees, with saline injected knees showing a slight reduction in response. Although the saline injected knees showed a trend towards rising sensitivity to adrenaline with time, none of the values differed significantly from the control response, whereas in the kaolin injected knees all the responses differed significantly from control $(\mathrm{p}<0.01)$. Six hours after kaolin injection the same dose of adrenaline produced about a $90 \%$ reduction in flow.

The differences in response to intra-arterial injection of adrenaline are illustrated well in fig 6 , which shows dose-response curves to adrenaline. Figure 6A shows that the response to adrenaline eight hours after injection of kaolin was enhanced compared with the response after saline injection after the same length of time. Figure 6B is from the same animal and indicates that the response to injected adrenaline was only little affected by time.

\section{Discussion}

The results of these experiments show that within eight hours of inducing an acute inflammatory response in the rabbit knee by intraarticular injection of $4 \%$ kaolin, changes occurred in the sensitivity of the $\alpha$ adrenoceptors. The response to close intra-arterial injection of adrenaline shows that postjunctional $\alpha$ adrenoceptors become more sensitive within two hours of kaolin injection and by six hours give rise to an additional $40 \%$ reduction in blood flow compared with the response to the same dose $\left(10^{-6} \mathrm{~mol} / \mathrm{l}\right)$ immediately after kaolin injection. The response to nerve stimulation might have been expected to show a similar increase in effect, but overall there was a smaller increase in the constrictor response (maximum increase about $19 \%$ ). This might be explained if the inflammatory process also enhances the sensitivity of prejunctional $\alpha$ adrenoceptors. These are usually of the $\alpha_{2}$ subtype and act in a negative feedback fashion, limiting the release of noradrenaline from sympathetic nerve endings. $^{6}$ Preliminary experiments suggest that such receptors are found in the rabbit knee and act as indicated above (Khoshbaten and Ferrell, unpublished observations). Another possibility is that the inflammatory process enhances the mechanisms involved in the reuptake of noradrenaline at symptomatic nerve endings and this contributes to the smaller enhancement 
of the constrictor response to nerve stimulation in kaolin injected knees.

It is noticeable that even in the saline injected knees a gradual increase in sensitivity to injected adrenaline occurred over eight hours (fig 5). This was not due to increased sensitivity of the $\alpha$ adrenoceptors to cold as cooling the knee artificially with a fan and repeating the administration of adrenaline failed to show increased sensitivity in the cooled, saline injected knee. This must therefore reflect some other factor acting to increase the sensitivity of $\alpha$ adrenoceptors. One possibility is that exposure of the capsule and insertion of thermocouple probes into the synovial cavity may provoke a low grade inflammatory response over the eight hour measurement period.

The dilator response which follows nerve stimulation showed a gradual rise in the kaolin injected knee with the value at $71 / 2$ hours having doubled compared with the control value. By contrast, in the saline injected knee the value at $71 / 2$ hours is only slightly increased compared with its control value. Previous work has shown that the dilator response is mediated by substance $P$ released from $C$ fibre afferents innervating the knee. ${ }^{2}$ The increased dilator response in the inflamed knee may reflect greater release of substance $P$ from these nerves fibres or perhaps upregulation of substance $P$ receptors. As substance $P$ is known to exert proinflammatory actions on joint tissue, ${ }^{5}$ increased release of substance $P$ or increased sensitivity of substance $\mathbf{P}$ receptors may contribute to the inflammatory process.

One hypothesis which was to be tested in these experiments was whether the vasodilatation which occurs in inflamed joints might be attributed to some extent to a decrease in $\alpha$ adrenoceptor sensitivity to noradrenaline release from sympathetic nerve endings on articular blood vessels. It is clear, however, that the opposite occurs-these become sensitised by the inflammatory process. This is mitigated to some extent by the possible sensitisation of prejunctional $\alpha$ adrenoceptors. An additional mitigating factor may be the release of substance $P$ from unmyelinated afferent fibres. These fibres show enhanced neural activity in acutely inflamed joints, ${ }^{7}$ and thus may contribute to vasodilatation in such joints.

These experiments have shown that acute joint inflammation induced by kaolin influences those factors which normally regulate articular blood vessel calibre. The mechanisms responsible for altering the sensitivity of $\alpha$ adrenoceptors (and perhaps substance $P$ receptors), and the functional significance of this, remain to be determined.

This research was supported by the MacFeat Bequest of the This research was supported by the MacFeat Bequest of the
University of Glasgow. The authors thank Dr F Y Lam for help University of Glasgow. The auth
with the Evans blue estimations.

1 Cobbold A F, Lewis $O \mathrm{~J}$. The nervous control of joint olood vessels. F Physiol (Lond) 1956; 133: 467-71.

2 Ferrell $W R$ Cant $R$. Vasodilation of articular blood vessels induced by antidromic stimulation of articular $C$ fibre afferents. In: Schmidt R F, Schaible H-G, Vahle-Hinz C, eds. Fine afferent fibres and pain. Weinheim: VCH, 1987: 187-92.

3 Ferrell W R, Khoshbaten A. Adrenoceptor profile of blood vessels in the knee joint of the rabbit. $\mathcal{F}$ Physiol (Lond) $1989 ; 414: 377-83$.

4 Geborek P, Forslind K, Wollheim F A. Direct assessment of synovial blood flow and its relation to induced hydrostatic pressure changes. Ann Rheum Dis 1989; 48: 281-6.

5 Lam F Y, Ferrell W R. Inhibition of carrageenan induced inflammation in the rat knee joint by substance $P$ antagonist. Ann Rheum Dis 1989; 48: 928-32.

6 Vizi E S. Presynaptic modulation of neurochemical transmission. Prog Neurobiol 1979; 12: 181-290.

7 Schaible H-G, Schmidt R F. Direct observation of the sensitization of articular afferents during an experimental arthritis. In: Dubner R, Gebhart G F, Bond M R, eds. Proceedings of the Vth world congress on pain. Amsterdam: Elsevier, 1988: 44-50. 